\title{
Analysis of Traditional Fur Processing Technology of Hui Nationality and Its Inheritance
}

\author{
Jianjun Ma \\ Institute of Hui Studies \\ Ningxia University \\ Yinchuan, China 750021
}

\begin{abstract}
The Hui people are good at business, which is an important part of their economic life. As a kind of business that Hui people are good at, the fur processing industry is a traditional craft that Hui people hold for thousands of years. It not only has a long history, a large number of employees and exquisite craftsmanship, but also plays an important role in the economic life of the Hui nationality, highlighting the Hui characteristics of business operation.
\end{abstract}

Keywords-Hui nationality; creative cultural industry; development; Yinchuan

\section{INTRODUCTION}

With a long history, the Hui fur processing industry is long-standing and well-established and has various forms and distinct national characteristics. The Hui fur processing industry is not only the traditional handicraft art of the Hui nationality, but also a window for people to know the Hui nationality's traditional character of being good at business. As a nation good at business, doing business constitutes an important part of the economic life of the Hui nationality. Hui business involves many industries, and has formed a certain scale, and in some industries it has a leading position. "This minority ethnic group often has a disproportionate economic power and a 'monopoly' in some industries, which should be paid special attention to when studying the history of Hui business." [1] For example, the fur trafficking and fur processing industries derived from the slaughtering industry, which has a "monopoly" status, all play an important role in the history of Hui commerce. Fur processing industry, as a business category that the Hui people are good at, is also a traditional craft that they have kept for thousands of years. It not only has a long history, but also is a characteristic category of Hui people's business. With a large number of employees, exquisite technology, this industry has an important position in the economic life of the Hui nationality, representing the Hui characteristics of business operation. By reviewing the history of the traditional fur processing of the Hui nationality and introducing its traditional skills, this paper aims at considering the traditional predicament and breakthrough of the traditional fur processing technology of Hui nationality at present.

\section{A COMMERCIAL SYMBOL Highlighting THE UNIQUENESS OF HUI ECONOMY AND CULTURE}

According to the origin of fur and the nationality it belongs to, the fur processing technology should be the livelihood and patent that nomads are good at, which not only conforms to the production and life reality of the nomads who mainly produce livestock products, but also accords with the cultural practice of the nomads' economic and cultural types. The fact is not so. Driven by commercial and economic interests, the Hui nationality has replaced the nomads who should be good at fur processing industry and continuously developed this industry into a highly developed fur processing industry. They thus rank the first among the minorities in northwest China in terms of fur processing, and this industry finally become a flourishing industry that is generally operated by the ethnic group. In the eyes of foreign countries, the Hui people in northwest China are "the only people who participate in the purchase of wool and some other grassland native products." [2] Hui nationality not only masters fur processing technology, but also inherits and carries forward this traditional technology. In fact, fur processing cannot be regarded as a pure traditional handicraft, through which the cultural adaptability of Hui nationality in China can be seen. This kind of adaptation has a strong advantage, which highlights the uniqueness of Hui economy and culture.

From the perspective of life, if a nation regards traditional fur processing as a need for survival, it will be difficult for this industry to develop into an industry that the whole nation is generally engaged in, let alone to create a handicraft. However, the Hui nationality generally practices it in the economic life, which even forms a unique commercial cultural symbol of the Hui nationality. The author considered the reasons why the fur processing of the non-nomadic Hui nationality was so prosperous. This is essentially related to the economic and cultural types of the Hui nationality. The Hui nationality is mainly engaged in agriculture, well as commerce and animal husbandry which constituting its means of livelihood. Therefore, some nomadic people with fur processing skills only have their skills remaining at the level of meeting their subsistence needs due to the lack of commercial impetus. On the contrary, the livelihood mode of the Hui nationality integrates farming, animal husbandry and commerce, and the 
traditional handicraft generated from farming and animal husbandry practices gives impetus to commercial operation. Since modern times, "Hui merchants have played an extremely important role in fur trade and once became a powerful force to promote the development of trade." [3]

Therefore, this is also the unique feature of the traditional fur processing technique of the Hui nationality, which shows the creative cultural practice of the Hui nationality and its effective use of natural resources. Through the inheritance and development of the traditional fur processing technology, the Hui nationality has brought it into full play. In the eyes of Hui people, the ordinary livestock are not only to meet people's meat needs, but also to be used as commodities for exchange. The livestock have use value all over their body, with meat to be eaten and fur to be used. This is both the premise of the existence of traditional fur processing technology and an important driving force for its sustainable development. This makes fur processing, as a traditional skill, a way to make a living. It is easy to become a business activity that produces economic benefits, and finally becomes the unique commercial symbol of Hui economy and culture.

\section{History OF THE TRADITIONAL FUR PROCESSING OF HUI}

The traditional fur craft of Hui has a long history with the rise of Hui slaughtering industry and fur trafficking. Especially since the Ming and Qing dynasties, the fur processing of the Hui people gradually prevailed, and formed a distinctive industry category, prevailing in the communities of the Hui people throughout the country. In some areas inhabited by Hui people, some Hui villages are engaged in fur processing as a whole, forming some exclusive fur processing Hui villages or some that took it as a sideline. The fur products they processed had exquisite style, were comfortable and beautiful, and were exported to all parts of the world, enjoying great popularity. During the Qing Dynasty, the Guanzhong region of Shaanxi province became the main settlement area of the Hui nationality in northwest China, and the fur processing industry took off in Guanzhong accordingly. At that time in the area of the Qiangbai town and Wangge village of Dali county, Dongfu, numerous people were engaged in fur processing industry. And this area became a fur production and distribution center at that time, and the fur products they processed sold well, some of which were exported to other places. Zhaotong of Yunnan province was an important place to enter Guizhou and Sichuan in Ming dynasty. The local Hui people mainly live in the southeast ridge there and they had always been making a living by rolling and making felts and making furs until the Qing dynasty. They processed a variety of furs with rich contents, mainly processing cowhide, horn and sheepskin into cowhide clothing and leather goods, etc. According the American James Milvard, "In China, where the wool trade flourished in the late 19th and early 20th centuries, many of them played an important role as middlemen... Later in 1910 and 1920, when the Beiyang Government was weak, the Hui merchants even controlled the entire Chinese wool trade like a central agency." [2] In the late Qing dynasty and the Republic of China, the Hui leather industry in Yunnan was once flourishing and wellknown. They take the form of individual management, with the family workshop as the production unit, throughout the urban and rural areas, producing and marketing all on their own. In the fur processing, they mainly adopt the method of nitre tanning, then steep the leather in the bark of cypress and Manchurian fir, and fumigate it with straw. The fur products are natural leather, red leather, curry leather and so on. The Jinniu Street in Kunming, was once a Hui fur processing street. At a glance, leather workshops in the whole street leap to the eyes. Due to the flourishing Hui leather industry, the streets are filled with the smell of tanned leather, so Jinniu Street is called "Smelly Leather Street".

At the same time, in the Hui communities in the north, fur processing was more prosperous. In addition to the Wangge village and Qaingbai town in Shaanxi province, Zhangjiachuan, Hezhou (Linxia Hui Autonomous Prefecture today) and Lanzhou in Gansu, Xining and Datong in Qinghai, Guyuan, Haiyuan, Tongxin and Wuzhong, etc. in Ningxia Hui Autonomous Region, Sangpo village in Mengxian county of Henan province, Jining in Shandong province and Beijing and Tianjin, etc. were all places where the Hui fur and leather processing industry developed. The fur processing in Jining, Shandong has a long history. Since the Ming dynasty, local Hui people started fur processing workshops. By the middle of the Qing dynasty, a production pattern of more than 70 fur processing workshops had been formed, in which the Ma Family Workshop was the most famous and has the largest scale. By the end of the Qing dynasty, the fur processing of the Hui nationality in Jining developed rapidly, and the number of large workshops in the area increased rapidly to more than 20 . Fur processing of Hui nationality in the Northeast appeared with the arrival of Hui people. It started late but developed rapidly. At the end of the Qing dynasty, a small number of Hui people began to be engaged in leather processing industry in Changchun, Jilin province. The first was the "Decheng" workshop started by a family surnamed Han in Yucheng, Sahndong province, followed by the Bai family in Hebei Province and Jin family in Shandong who came to Changchun for fur processing industry, etc. By 1932, there were more than 20 leather shops opened by Hui people in Changchun, like the "Defayong" in Sandao Street (Dongmen li) set up by the brothers of Jin Chunyuan, Jin Chunlian From the Jinzhuang village of Yucheng county, Shandong province, the "Sanhecheng" in the Linhe Street set up by Sha Enzhong, a man surnamed Han and another surnamed Zhao from Pingyuan county, Shandong province, the "Leather Shop of the Family Shi" in the Sidao Street set up by Shi Chaogong" from Enxian county, Shandong province, the "Leather Shop of the Family $\mathrm{Ma} "$ in a street about the Erdaohezi (Erdaohe district today, namely there were two rivers there" set up by a Hui family surnamed Ma from Dayingzi village of Shuangyang sounty in Jilin province, the "Yongyuanhe", "Fuguihe" and "Yongshengmao" in the Sandao Street set up by Yang Zhangyuan and his two relatives from Yangxin county, Shandong province, etc., accounting for one fifth of the leather trade in Changchun. The leather shops set up by Hui people are relatively concentrated, mainly in the areas of 
Changsandao Street, Dajing Road, etc., for mutual support. [4]

\section{ThE TRADITIONAL TECHNIQUE OF FUR PROCESSING OF HUI NATIONALITY}

The Hui nationality is an ethnic group with a wide population distribution. The Hui people live scattered across the country on the whole and in compact communities in each place, which makes it strongly heterogeneous inside this group. In particular, the traditional fur processing, as a traditional national handicraft of Hui, has formed a rich variety of processing techniques due to the regional differences. The Hui nationality distributed in different areas have their own unique innovation in the inheritance of traditional fur processing technology, and finally formed the traditional fur processing technologies of Hui nationality with regional characteristics. For example, in history, the Hui people in Dali county, Shaanxi were good at tanning leather with nitre, while Hui people in Qinghai province mainly tanned leather with well-proportioned nitre, salt and flour, and Hui people in Jinan, Shandong took nitre and glutinous rice as the materials for tanning, etc.

In the specific processing and production process, especially in this key process of tanning, it was largely identical but with minor differences in different regions and has diverse styles. For example, more than 20 processes were required in fur processing of Hui people in Qinghai province, the process of which was complex and strongly technical. In general, the processes of sheepskin air drying, curing with salt, primary roughening, cleaning, second roughening, curing in vat, primary meat and oil removal, second cleaning, tanning, tinting, drying in the sun, moisture regain, elaborate processing, mellowing and sorting out the fur, etc. are required [5]. In the tanning part of fur processing Hui people in Jinan, Shandong, 20 processes are required including steeping, oil dirt removal, cleaning, treading down, sinking in vat, moving out of vat, water controlling, drying in the sun, scraping and hair taking, etc. [6]. In order to show the traditional technology of fur processing of Hui nationality, the author takes the traditional kivskin processing technology in Ningxia as an example and depicts the traditional kivskin processing technology of Hui nationality in Ningxia through field work, so as to peek into the rich connotation of the traditional fur processing technology of Hui nationality.

The Hui nationality in Ningxia Hui Autonomous Region has a traditional habit of processing fur that is used to make kivskin clothing and waistcoat, etc., well- received by the Hui people in Ningxia. Fur processing technology prevails in Hui communities including Yanchi, Haiyuan, Tongxin, Wuzhong, Yongning, etc. in Ningxia. The Hui traditional fur processing technology in Ningxia has its own characteristics and there are many unique techniques in the links of leather selection and tannage. The fine and exquisite workmanship can be seen in the specific processing process. In general, the major processes include leather selection, tannage and manufacture, etc.

The first step is leather selection. The Hui people in Ningxia attach great importance to the materials of fur processing, which is also their most prominent feature. The materials are mostly kivskin taken from Tan Sheep (a kind of sheep with long-staple wool raised in Ningxia and Gansu) of Ningxia about 30 days after the birth of the lamb. As the local Hui people said, the fur taken after the lamb has enough fur about 30 days after birth is kivskin after is processed. Local Hui craftsmen especially like the fur of lambs born in winter. Ningxia Tan Sheep is a kind of sheep that grows in Ningxia. Its uniqueness lies in that it has fat tail and coarse wool, which is unique in the world. The kivskin made from Ningxia Tan Sheep is thin as Thick paper, strong but pliable, soft and uniform in texture and the hair is naturally tufted, curving and soft. So it is an excellent natural fur material.

The second step is tanning. Tanning is an important processing procedure of kivskin and the core of traditional fur processing technology of Hui nationality in Ningxia. This procedure is operated by a special craftsman and can be done in either season of the year. The required conditions are different in different seasons. In winter, the tanning has to be made in a vat on fire, while in other seasons this process can go in the natural temperature. The materials required in making kivskin are characteristic. The ingredients are mainly rice stone or coarse rice, with nitre and salt as supplement. The ingredients should be properly distributed, usually $70 \%$ to $80 \%$ of rice stone and $20 \%$ to $30 \%$ of nitre or salt. Then the kivskin is put into the vat for tanning. The tanning process is complex, yet very typical. Thus the craftsmen generally pay the most attention to this process and value it very much. In fact, if the craftsman is not skillful enough, the leather he makes will tend to breed pest, discolour, shed hair or even stink. After the tanning process, the leather is taken out of the vat and dried in the sun, after which it will be pulled and spaded

The last step is manufacture. At this time, the leather is already tanned, and it can be used for manufacture after a few times of cleansing. The next step is to take a "Four Shallow Nest Leather (the leather of four joints connecting sheep's belly and legs)" and nail it on the plank with its fur facing inside and the other side dipped with water. Effort should be made at this time to widen it while knocking in nails. After getting dried, the leather is washed with acacia water until "bending" appears. Then after being pressed with planks it can be sowed with precision work, thus a kivskin good is finished.

\section{THE INHERITANCE OF HUI TRADITIONAL FUR PROCESSING TECHNOLOGY AND ITS PREDICAMENT}

Traditional fur processing techniques enrich the folk handicraft art of the Hui nationality with the most common presence in the Hui society, highlighting the unique artistic creativity of the Hui nationality. The Hui fur processing craftsmen put it into the art of living and they are both creators and inheritors of fur processing technology. In the intergenerational teaching of transmission from father to son or from master to apprentice, skills are passed on from generation to generation. The inheritance of this craft from the ancient times to today should give the credit to generations of traditional fur-makers. As both craftsmen and traders, making a living makes them be passionate for the fur 
processing technology, and their dedication to art gives their labor and creation the source of living water, so they are not afraid of the hardships of fur processing and are never bored in the pursuit of this traditional handicraft.

Since modern times, there have been more Hui people engaged in fur processing as a business, so that the Hui fur processing industry enjoys prosperity. Due to its large number of employees and wide distribution, it has become an important part of traditional Hui business. While showing the characteristics of Hui business structure, it contributes to the formation of one of the "industry monopoly" phenomena of Hui business economy historically [1], which is a dominant cultural symbol of Hui business. Sangpo, a Hui village in Henan with just thousands of people, is an example. Due to its prosperous fur and leather processing industry and large number of personnel engaged in this trade, the Sangpo village has seen almost all of its villagers occupied in fur processing and its products being sold to big cities in the country. Today, the Hui fur processing has realized the transformation from traditional small workshop to modern factory. There are more than 130 fur processing enterprises, processing 15 million pieces sheepskin per year with up to 10,000 workers, and the products are sold overseas. With objective economic benefits, Sangpo enjoys the reputation of "Fur Capital of China". There are over 60 thousand people engaged in the fur processing industry in Zhangjiachuan Hui Autonomous County in Gansu. More than 6 million pieces of fur are sold across the country, with a turnover of 100 million yuan. Its production of sheepskin coat, leather jackets and so on not only sell well at home, but are also exported overseas. The XiangsHui village is a Hui vilage of Haiya township in Haiyuan county, Ningxia. Since the 1980s, the whole village have been engaged in fur processing, and now both men and women in the village do fur work, and every family does fur processing. The bedspread, mattress, vest, scarf, shawl, kneepad and so on they produced are delicately made and show elegant appearance, thus are sold at home and abroad and deeply loved by consumers. Now this village is a genuine professional kivskin processing village in Ningxia.

With the development of the Hui fur processing industry, the inheritance of the traditional Hui fur handicraft is also confronted with the dilemma of the times. In fact, in the transition from traditional society to modern society, the universal presence of modernity in the field of traditional life has made the traditional technology enter the era of industrialization in the modern transformation and as a result, the soil in which traditional skills survive is lost. Besides, when machines take the place of manual labor, the artistry of traditional crafts is greatly diminished in mechanized and mass efficient commodity production, which is the dilemma of the times in the modern transformation of all traditional crafts. Someone has pointed out: "the advanced productivity of the modern industrial civilization makes industrial products enter the large-scale 'integrated' batch production process, greatly reducing the production cost. Therefore, in pursuit of higher and more effective income, profit-oriented businessmen can only make the time-consuming and 'fine' traditional handicraft take a back seat before modernization and replaced by the cheap modern crafts with the same function. [7] The contradiction interwoven in the modern transformation of traditional craft has become a realistic problem that must be faced. In the transformation and upgrading of traditional manual production to modern factories, on the one hand, there are fewer traditional fur processing workshops. For example, there remain less than ten kivskin processing workshops in Tongxin County, where there used to be 50; the 800 in Haiyuan County is reduced to about 50 . On the other hand, while increasing the efficiency, the industrial manufacturing with assembly lines where machines take the place of men, weakens the artistry of traditional furs and furs as handicraft itself, and also causes the lack of successors of the inheritors of traditional skills. With the departure of some old artists, this situation is becoming more and more obvious, and the dilemma of the inheritance of traditional skills is presented. How to inherit the Hui traditional fur crafts has become a realistic problem.

\section{The SOlution to THE CURRENT DilemMA OF ART INHERITANCE}

The plight of the inheritance of traditional fur processing technology is not unsolvable. Actually, the presence of this problem is inevitable with the development of the times. The protection and inheritance of traditional skills in the era of intense modernization, globalization and economic integration is a realistic problem facing mankind. This requires people to have a correct understanding from concept. While taking it as a social norm, people should actively seek for protection strategies, especially innovation in traditional ways, so as to realize the continuous inheritance of Hui fur processing technology in the new era. Here, the author holds that three things should be done.

\section{A. Integrating Traditional Crafts into Local Vocational and Technical Education System}

Traditional folk craft is in the form of individual family workshop that limits the inheritance of traditional skills to individual people, so that the traditional craft is only transmitted across generation from individual to individual through the mode of family inheritance and apprentice inheritance. In this way, the skill inheritance is not accessible to the public. Since this mode of inheritance tends to break the chain of inheritors, the popular mode of school education should be adopted. The traditional fur processing technology should be incorporated into the local vocational education system, so that the famous folk craft can go to the classroom, thus achieving a breakthrough in the form of technology inheritance and talent training. It is suggested that vocational and technical school for nationalities with available conditions should set up school-based teaching materials for traditional arts and crafts of the Hui nationality and employ local traditional artists to teach the courses. For the majority of vocational education students, or those interested in the folk craft, focused training, various forms of lectures and training on Hui folk craft can be arranged to bring the folk craft masters to the classroom, so as to let more people master this traditional craft. 


\section{REFERENCES}

B. Stimulating the Enthusiasm of the Hui People to Inherit the Fur Processing Technology Through Promoting the Economic Benefits

Inheriting craft is both a kind of human art, and a way for artists to obtain survival materials. For artists, the reason why traditional crafts can be inherited from generation to generation is that the skills can create a certain economic value for them or maximize the economic value of traditional skills. Hui traditional fur processing technology can survive widely, because there is always a certain market demand for it, and it create a certain economic value as a commodity. This determines that in order to pass on the Hui fur processing technology, it is important to expand the market demand and improve its economic effect, so as to really promote Hui people's initiative of inheriting the fur processing technology. Just as someone has said, "Economic benefits are one of the most important levers for the inheritance and development of a traditional craft. Only by expanding the market and increasing the economic benefits of traditional craft can the passion for protection and inheritance be aroused." [7]

\section{Stimulating the Subjectivity and Consciousness of Local People in the Inheritance of Hui Traditional Fur Processing Technology}

To realize the inheritance of Hui traditional fur processing technology, on the one hand, efforts should be made to stimulate the subject consciousness of local Hui people and enhance their sense of mission and responsibility of inheriting their own culture through propaganda and education. On the other hand, it is necessary to make the Hui people take the initiative to participate in the inheritance and protection of the traditional fur processing technology, so that inheritance and protection can become a conscious act. Especially for the Hui families engaged in fur processing, it is important to achieve the inter-generational transmission of skills, and not to give up the responsibility of the inheritance of skills because of economic interests and other reasons. They should strengthen the sense of responsibility, and strive to do a good job in the inheritance and development of traditional skills.

\section{CONCLUSION}

In the process of transformation from traditional society to modern society, the importance and urgency for the fur processing technology, which is one of the symbolic intangible cultural heritages of Hui nationality to realize its own sustainable development, are self-evident, in the face of the fierce impact of modernization. Under the new historical conditions, it is necessary not only to safeguard traditions, but also to make innovations in technology and other aspects. This is not only the solution to solving the current dilemma, but also the inevitable requirement for the technology to better adapt to social development.
[1] Ma Ping. Analysis of the "industry monopoly" problem of modern Hui economy [J]. Researches on the Hui, 2002 (1). (in Chinese)

[2] (US) James Milvard. Wool trade between Hui Merchants and Chinese Border Areas from 1880 to 1909 [J]. Translated by Li Zhankui. Ethno-National Studies in Gansu, 1989(4). (in Chinese)

[3] Zhong Yinmei. Hui Merchants of Modern Fur Trade in Gan-NingQing Areas [J]. Journal of Hui Muslim Minority Study, 2009 (4). (in Chinese)

[4] Fur Processing Industry of Changchun Hui People in the Period of Manchukuo [N]. Changchun Evening News, 2013-12-30. (in Chinese)

[5] Ma Cong. The Last Cobbler - an Old Man of Hui Nationality in Xining Who Made Leather by Hand [J/OL]. Muslim Online, 201404-04. (in Chinese)

[6] Yuan Zhouwei. Production and Sales Network of Hui Furs in Modern Times [J]. Journal of Hui Muslim Minority Study, 2008 (3). (in Chinese)

[7] Jiang Ting. Modern Dilemma and Solution of Traditional Handicraft a Case Study of Dongguan Street in Yangzhou [J]. Theoretical Exploration, 2013 (9). (in Chinese)

[8] Wei Danfang. Inheritance Dilemma and Countermeasures of Traditional Technology - a Case Study of Sandpaper Technology in Gongchuan, Guangxi [J]. Journal of Guangxi University for Nationalities, 2007 (4). (in Chinese) 